\title{
Plasma microRNA-19a as a potential biomarker for esophageal squamous cell carcinoma diagnosis and prognosis
}

\begin{abstract}
Aim: To investigate whether plasma miR-19a can serve as a biomarker for esophageal squamous cell carcinoma (ESCC) diagnosis and prognosis. Materials \& methods: Plasma samples from $89 \mathrm{ESCC}, 45$ benign lesion patients and 80 healthy controls were subjected to RT-qPCR analyses for miR-19a. In addition, plasma samples from 30 patients were collected before and after surgery for the same analyses. Results: Plasma miR-19a was significantly increased in ESCC patients compared with healthy controls. The sensitivity of $m i R-19 a$ for early stages of ESCC was $68.09 \%$. Combination of $m i R-19 a$ and cytokeratin 19 fragment 21-1 (Cyfra21-1) further improved the sensitivity to $78.70 \%$. Moreover, plasma miR-19a level was decreased in patients after surgery. Conclusion: Plasma miR-19a may serve as a potential biomarker that complements Cyfra21-1 in detecting early stages of ESCC.
\end{abstract}

First draft submitted: 13 October 2016; Accepted for publication: 13 February 2017; Published online: 16 June 2017

Keywords: biomarker $\bullet$ Cyfra21-1 • esophageal squamous cell carcinoma $\bullet$ miR-19a - plasma

Esophageal cancer (EC) is one of the leading aggressive malignancies worldwide and is the fourth leading cause of cancer-related deaths in China [1]. The incidence of high-risk EC in China even exceeds 130 per 100,000 individuals $[2,3]$. The major pathological type of $\mathrm{EC}$ is esophageal squamous cell carcinoma (ESCC), which has been most frequently identified in Northern Iran and NorthCentral China [4]. Although there has been significant improvement in diagnosis and treatment, the overall 5-year survival rate remains only about $25-30 \%$ for patients who receive curative surgery $[5,6]$, while the rate dropped to $13 \%$ for patients with lymph node metastasis [7]. Early detection is critical for improving outcomes and reducing mortality of ESCC patients.

Although biopsy and imaging examination have improved the detection rate of ESCC, these methods are invasive or require radiation, greatly limiting their applications $[8,9]$.
Currently, traditional tumor markers, such as cytokeratin 19 fragment (Cyfra) 21-1 and squamous cell carcinoma antigen, are used to diagnose and evaluate ESCC progression. However, they both exhibit a low sensitivity. Yamamoto reported that the sensitivity of Cyfra21-1 for the diagnosis of ESCC was only $47.9 \%$ [10]. Mealy showed that the sensitivity of squamous cell carcinoma antigen was about $32 \%$ [11]. The absence of sufficiently sensitive biomarkers limits early diagnosis. Therefore, there is an urgent need to identify novel, easy-to-assay biomarkers for early diagnosis and prognosis of ESCC to improve outcomes and reduce mortality of ESCC patients.

miRNAs are small noncoding RNAs of 21-25 nucleotides in length that negatively regulate target genes [12] and play important roles in a wide range of physiological and pathological processes [13,14]. Genome-wide studies have demonstrated that miRNA genes
Yongying Bai ${ }^{\ddagger 1,13}$, Huayue Lin ${ }^{\ddagger 1}$, Zanxi Fang ${ }^{1}$, Qing Luo ${ }^{1,2}$ Yizhen Fang', Yuanhui Su', Qing $\mathrm{Hu}^{1}$, Hongbing Duan ${ }^{4}$, Falin Chen $*, 5$ \& Zhong-Ying Zhang $* *, 1,2$

${ }^{1}$ Center for Clinical Laboratory, Xiamen University Affiliated Zhongshan Hospital, Xiamen, China

${ }^{2}$ State Key Laboratory of Molecular Vaccinology \& Molecular Diagnostics, School of Public Health, Xiamen University, Xiamen, China ${ }^{3}$ Clinical Analysis Center, Women's Hospital, School of Medicine, Zhejiang University, Hangzhou, China ${ }^{4}$ Department of Thoracic Surgery, Xiamen University Affiliated Zhongshan Hospital, Xiamen, China

${ }^{5}$ Clinical Laboratory Center, Fujian Provincial Hospital, Fuzhou, China *Author for correspondence: falinchen@126.com

**Author for correspondence: zhangzy1121@xmu.edu.cn ${ }^{\ddagger}$ Authors contributed equally 
are frequently located in cancer-associated genomic regions or in fragile sites, indicating that the potential great roles in tumorigenesis [15]. Since their discovery in 1993, emerging evidence shows that altered abundances of miRNAs are associated with cancer, such as ESCC [16-18], liver cancer [19-21] and breast cancer [22-24]. miRNAs are detectable in plasma in a remarkably stable form that is often resistant to RNase digestions [25], making circulating miRNAs ideal candidates to serve as biomarkers for cancer detection. However, there have been few reports to identify suitable circulating miRNAs for diagnosis, prognosis and recurrence/metastasis prediction of ESCC.

Previous studies showed that miR-19a acted as an oncogene in ESCC. It promotes cellular growth in vitro and in vivo and reduces apoptosis [26]. Compared with levels in the surrounding noncancer esophageal tissue, $m i R-19 a$ was elevated in ESCC. Additionally, the overexpression of miR-19a is an independent prognostic factor for overall survival and progression-free survival in ESCC [16]. Although the role of miR-19a as an oncogenic miRNA in cancer cells and/or tissues has been relatively thoroughly studied, the potential value of this miRNA as a circulating biomarker for the detection and prognosis for cancer, especially for ESCC, has not been studied. In this report, we found that plasma miR-19a was elevated in patients with ESCC and downregulated in patients after surgery. Thus, the plasma level of miR-19a has promising potential to serve as a novel biomarker for ESCC diagnosis and prognosis and further efforts to develop miR-19a as a biomarker for ESCC diagnosis and prognosis are needed.

\section{Materials \& methods}

\section{Ethics statement}

The study was carried out according to the ethical principles of the 2008 revised Declaration of Helsinki. All plasma-based studies were approved by the Ethics Committee of the Xiamen University affiliated Zhongshan Hospital. All participants gave a written consent and agreed their information to be stored in the hospital database and used for research purposes.

\section{Plasma sample collection}

Between July 2013 and December 2014, blood samples from 89 ESCC and 45 benign lesion patients (14 esophageal intraepithelial neoplasia, 17 esophageal leiomyomata, 2 esophageal polyps and 12 esophagitis) receiving treatment at Xiamen University affiliated Zhongshan Hospital were collected. In addition, 80 age- and gender-matched blood samples from healthy individuals with no history of cancer and in good health on the basis of self-report as well as with normal physical examination were collected. The exclusion criteria of blood samples were as follows: plasma from patients who were diagnosed with other digestive tract disease; plasma from patients who received operational treatments, chemotherapy or radiotherapy treatment before collection; plasma from patients whose pathological data were not complete; plasma was hemolytic or turbid. The blood samples were collected from patients before operational treatments, chemotherapy or radiotherapy. At 10-12 days post operation, paired-plasma samples were collected from 30 patients. All plasma samples were centrifuged as described previously [27] and then the plasma was divided into aliquots, following snap freezing at $-80^{\circ} \mathrm{C}$. Clinical characteristics of ESCC are summarized in Table 1.

\section{MicroRNA isolation}

miRNA was isolated with miRcute miRNA extraction kit (TIANGEN, Beijing, China) according to the manufacturer's instructions. A synthetic miRNA cel-miR-39 (Qiagen, Dusseldorf, Germany) was added to each $200 \mu \mathrm{l}$ plasma specimen at a final concentration of $5 \mathrm{nM}$ as a reference gene before isolation. The extracted miRNA was eluted in $30 \mu \mathrm{l}$ RNase-free water (TIANGEN) at a concentration ranging from $5-50 \mathrm{ng} / \mu \mathrm{l}$. The absorbance values of $\mathrm{OD}_{260} / \mathrm{OD}_{280}$ were between 1.8 and 2.1. All isolated miRNA samples were quickly aliquoted and immediately stored at $-80^{\circ} \mathrm{C}$ until use.

\section{CDNA synthesis}

The reverse transcription system was $20 \mu$, containing $0.2 \mu \mathrm{l}$ Moloney murine leukemia virus (MMLV) reverse transcriptase $(200 \mathrm{U} / \mu \mathrm{l})$ (Promega, WI, USA), $0.2 \mu \mathrm{l}$ ribonuclease inhibitor $(40 \mathrm{U} / \mu \mathrm{l})$ (TAKARA, Dalian, China), $0.8 \mu \mathrm{l} 10 \mathrm{mM}$ dNTP mix (TAKARA), $1.2 \mu \mathrm{l}$ $10 \mathrm{mM}$ stem loop RT primer (SANGON, Shanghai, China), $2 \mu$ lemplate miRNA, $4 \mu$ l MMLV RT buffer (Promega, WI, USA) and $11.6 \mu$ l RNAase-free water (Promega). No-template controls for reverse transcription step were used to ensure target specific amplification. Reaction were performed at $25^{\circ} \mathrm{C}$ for $5 \mathrm{~min}$, $40^{\circ} \mathrm{C}$ for $60 \mathrm{~min}$ and finally at $70^{\circ} \mathrm{C}$ for $15 \mathrm{~min}$. The reverse transcription primers were as follows: $m i R-19 a$, 5'- GTCGTATCCAGTGCGTGTCGTGGAGTCGGCAATTGCACTGGATACGACTCAGTTT-3'; cel-miR-39, 5'-GTCGTATCCAGTGCGTGTCGTGGAGTCGGCAATTGCACTGGATACGACCAAGCTGA-3'.

\section{Reverse transcription quantitative real-time PCR}

The $20 \mu \mathrm{l}$ reaction mixtures for qPCR were carried out following the manufacturer's protocol of SYBR Premix Ex Taq TM II reagents (Tli RNaseH Plus from TAKARA). The PCR mixture $(20 \mu \mathrm{l})$ contains $10 \mu \mathrm{l}$ 
Table 1. Clinicopathological characteristics of 89 esophageal squamous cell carcinoma patients.

\begin{tabular}{|c|c|}
\hline Variables & n (\%) \\
\hline \multicolumn{2}{|l|}{ Gender: } \\
\hline Male & $69(77.53 \%)$ \\
\hline Female & $20(22.47 \%)$ \\
\hline Age & $58(43-81)$ \\
\hline \multicolumn{2}{|l|}{ Tumor grade: } \\
\hline $1+\|$ & $47(52.81 \%)$ \\
\hline $\mathrm{III}+\mathrm{IV}$ & $42(47.19 \%)$ \\
\hline \multicolumn{2}{|l|}{ T category: } \\
\hline 1 & $14(15.73 \%)$ \\
\hline 2 & $15(16.85 \%)$ \\
\hline 3 & $42(47.19 \%)$ \\
\hline 4 & $18(20.22 \%)$ \\
\hline \multicolumn{2}{|l|}{$\mathrm{N}$ category: } \\
\hline No & $38(42.70 \%)$ \\
\hline N1 & $51(57.30 \%)$ \\
\hline \multicolumn{2}{|l|}{ M category: } \\
\hline Mo & $87(97.75 \%)$ \\
\hline M1 & $2(2.25 \%)$ \\
\hline \multicolumn{2}{|l|}{ Differentiation: } \\
\hline High & $3(3.37 \%)$ \\
\hline Middle & $71(79.78 \%)$ \\
\hline Low & $13(14.61)$ \\
\hline Unknown & $2(2.25 \%)$ \\
\hline \multicolumn{2}{|l|}{ Location: } \\
\hline Up & $16(17.98 \%)$ \\
\hline Middle & $51(57.30 \%)$ \\
\hline Down & $21(23.60 \%)$ \\
\hline Unknown & $1(1.12 \%)$ \\
\hline \multicolumn{2}{|c|}{ Recurrence/metastasis after surgery ${ }^{+}$: } \\
\hline Non & $43(87.75 \%)$ \\
\hline Recurrence/metastasis & $6(12.24 \%)$ \\
\hline
\end{tabular}

SYBR mix, $6.8 \mu \mathrm{l}$ RNAase-free water, $2 \mu \mathrm{cDNA}$, $0.4 \mu \mathrm{l}$ ROX Reference Dye II, $0.4 \mu \mathrm{l} 10 \mathrm{nM}$ forward primer (SANGON) and $0.4 \mu \mathrm{l} 10 \mathrm{nM}$ reverse primer (SANGON). Each reaction was repeated twice. Notemplate controls for qPCR step were used to ensure target specific amplification. RT-qPCR was performed on ABI7500 (Applied Biosystems, Singapore) under the following reaction condition: $95^{\circ} \mathrm{C}$ for $30 \mathrm{~s}$, followed by 40 cycles: $95^{\circ} \mathrm{C}$ for $5 \mathrm{~s}$ and $60^{\circ} \mathrm{C}$ for $34 \mathrm{~s}$. The primers were as follows: miR-19a, 5'-TGGTGTGTGCAAATCTATGCA-3' (forward), 5'-CAGT-
GCGTGTCGTTGGAGT-3' (reverse); cel-miR-39, 5'-CAGAGTCACCGGGTGTAAAT-3' (forward), 5'-CCAGTGCGTGTCGTGGAGTC-3' (reverse). The expression level of miR-19a was normalized to $c e l-$ miR-39 and was calculated by using the $2^{-\Delta \Delta C_{q}}$ method. Then, the data were transformed to $\log _{10}$ for analyses.

\section{Biochemical analyses}

The plasma concentration of Cyfra21-1 was measured by Roche high-sensitivity assay performed on the Cobas e601 system based on the principle of 
electrochemical luminescence. The cut-off point of Cyfra21-1 is $3.39 \mathrm{ng} / \mathrm{ml}$ and the detection limit is 0.1 $\mathrm{ng} / \mathrm{ml}$ with a CV of $<5 \%$. Samples were randomized for testing and blinded to the trained clinical laboratory technician before interpretation.

\section{Statistical analyses}

The nonparametric Mann-Whitney U test was used to analyze miR-19a abundances in two groups and Kruskal-Wallis test was used in more than two groups. Wilcoxon signed-rank test was used to determine the relative expression between pre- and postoperation. Receiver operating characteristic (ROC) curves were applied to analyze the diagnostic values of miR-19a and Cyfra211. Youden Index (sensitivity + specificity-1) was used to identify the optimal cut-off threshold value. Logistic regression, serial testing and parallel testing were used in combination diagnosis of miR-19a and Cyfra21-1. The goal of logistic regression is to find the best fitting model to distinguish between the two groups. Serial testing is to improve specificity at the cost of lower sensitivity and parallel testing is to achieve higher sensitivity but lower specificity. The statistical analyses were carried out with IBM SPSS 19.0 software. The graphs were generated by using GraphPad Prism 5.0.

\section{Results}

\section{ESCC patients have increased abundance of} miR-19a in the plasma

Plasma miRNAs remain stable for a long period of time even under harsh conditions. For accurate assessment of the abundance of miR-19a in plasma samples, we first confirmed the stability of miR-19a in the

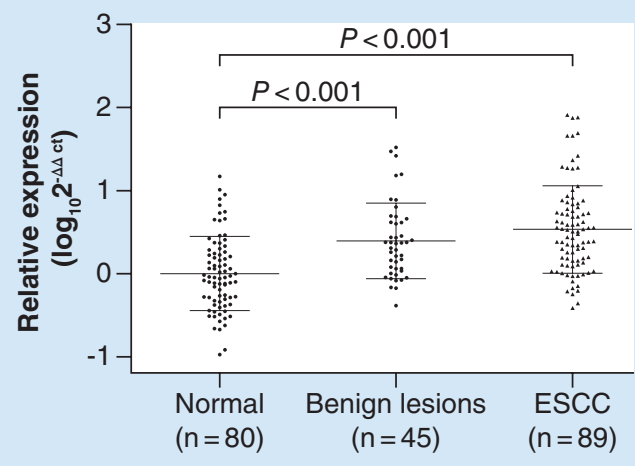

Figure 1. Plasma levels of $m i R-19 a$ were increased in patients with ESCC. The relative levels of miR-19a in patients with benign lesions, ESCC and healthy controls. All data were normalized to reference gene cel-miR-39 and shown as $\log _{10}$. The Kruskal-Wallis tests were performed to examine the difference of $m i R-19 a$ between normal controls and diseased groups. ESCC: Esophageal squamous cell carcinoma. plasma (Supplementary Figure 1). Incubation of the plasma samples at $4^{\circ} \mathrm{C}$ or room temperature $\left(22^{\circ} \mathrm{C}\right)$ for up to 7 days or repeated freezing and thawing at least five-times did not cause significant changes in quantification cycle (Cq value), indicating that $m i R$ $19 a$ was stable in the plasma and indicating that circulating miR-19a is an excellent candidate to serve as a biomarker for ESCC detection.

To assess plasma concentrations of miR-19a in patients with different conditions of esophageal disease, we compared the plasma levels of miR-19a between normal controls, patients with benign lesions and the ESCC group. As shown in Figure 1, the abundance of $m i R-19 a$ in the plasma was significantly increased in patients with benign lesions ( $\mathrm{p}<0.001)$ and ESCC (p $<0.001)$. Although there was no difference between benign lesion and ESCC groups ( $\mathrm{p}=0.572)$, there was a gradual uptrend of the miR-19a level from normal benign lesions - carcinoma.

The plasma level of miR-19a has diagnosis value for ESCC, early stages of ESCC and esophageal tumor

To determine whether the plasma level of miR-19a had ESCC diagnostic value, the ROC curve was applied to analyze sensitivity and specificity and the Youden Index was used to select the optimal cutoff. The area under the ROC curve (area under the curve (AUC)) for miR-19a in ESCC and noncancer controls ranged from 0.643 to 0.780 in the validation cohort of 89 patients and 125 noncancer controls (Figure 2A). With an optimal cutoff (0.2909) acc ording to the Youden Index, at which the sum of sensitivity and specificity was the maximal, the sensitivity and specificity for ESCC was 66.29 and $66.40 \%$, respectively (Table 2).

The level of Cyfra21-1 is commonly used as a diagnosis marker for ESCC. Therefore, we next compared the performance of miR-19a and Cyfra21-1 in the detection of ESCC. As shown in Figure 2B, Cyfra21-1 showed a lower AUC (0.676) than miR-19a (0.712). Although the positive predictive value (PPV) of $m i R$ $19 a$ was similar to Cyfra21-1, the negative predictive value (NPV), diagnosis efficiency and the sensitivity of miR-19a were higher than those for Cyfra21-1 (Table 2). These findings validate the performance of $m i R-19 a$ as a plasma marker for ESCC detection.

Early diagnosis and treatment of ESCC is of great value to improve the survival of ESCC patients. We next determined whether the plasma level of miR-19a can be used for early detection of ESCC (I + II). As shown in Figure $2 \mathrm{C}$, the AUC for early stages of ESCC and noncancer controls was 0.729 (95\% CI: 0.6480.810). At a threshold of 0.2909 , the sensitivity and specificity of miR-19a were 68.09 and $66.40 \%$ in dis- 
(A)

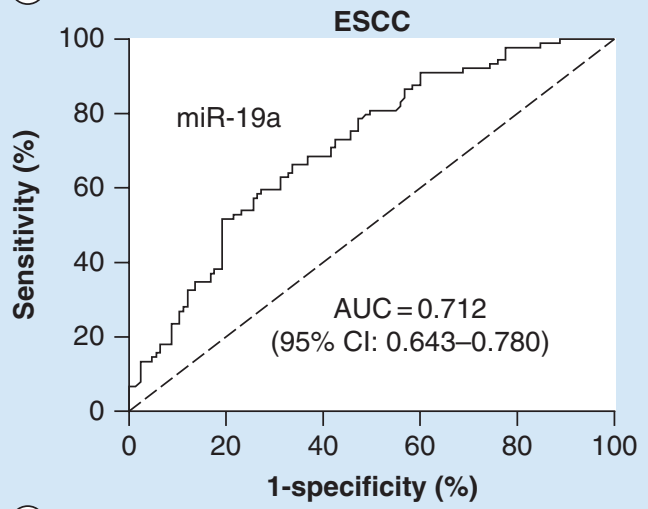

(C)

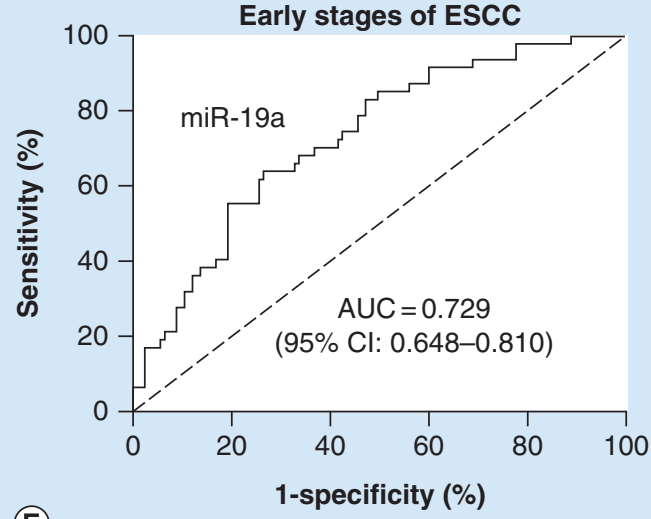

(E)

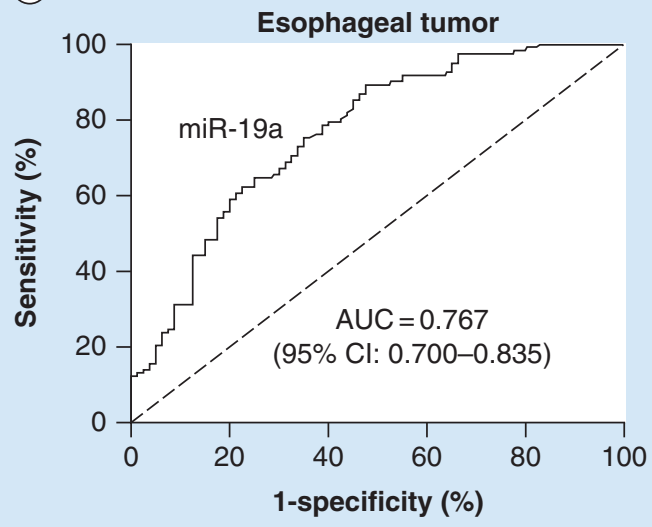

(B)

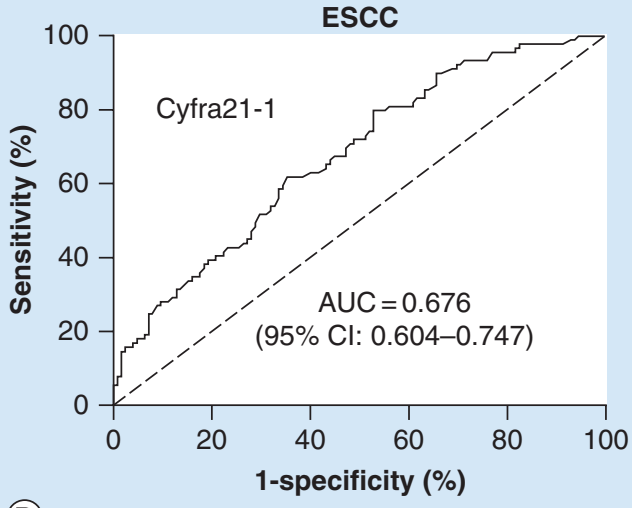

(D)

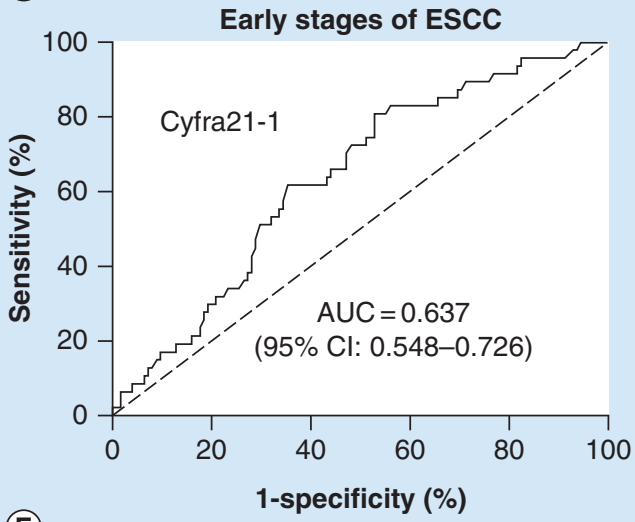

(F)

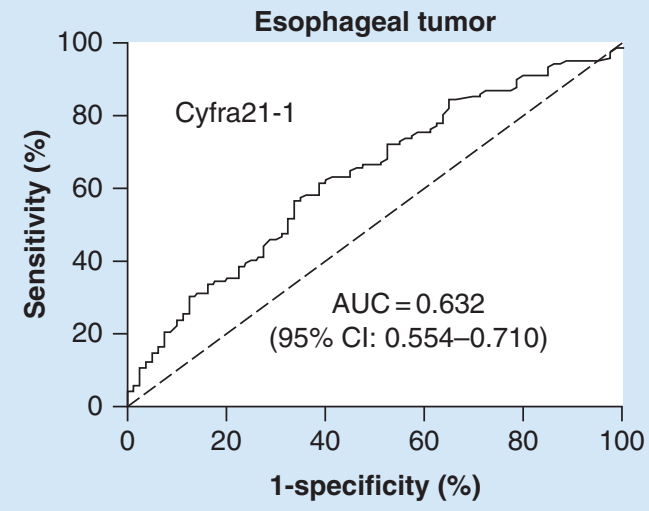

Figure 2. ROC curve analysis for detection of ESCC, early stages of ESCC and esophageal tumor. (A) ROC curves for miR-19a in 89 patients with ESCC and 125 noncancer controls. (B) ROC curves for Cyfra21-1 in 89 patients with ESCC and 125 noncancer controls. (C), ROC curve for miR-19a in 47 patients with ESCC (I + II) and 125 noncancer controls. (D) ROC curve for Cyfra21-1 in 47 patients with ESCC (I + II) and 125 noncancer controls. (E) ROC curve for miR-19a in 122 patients with esophageal tumor and 80 healthy controls. (F) ROC curve for Cyfra21-1 in 122 patients with esophageal tumor and 80 healthy controls.

Cyfra: Cytokeratin 19 fragment; ESCC: Esophageal squamous cell carcinoma; ROC: Receiver operating characteristic.

tinguishing early stages of ESCC from noncancer controls (Table 2). Consistently, the AUC, sensitivity, PPV and NPV for Cyfra21-1 are much lower than those for $m i R-19 a$ in the diagnosis of early stages of ESCC (Figure 2D \& Table 2). These findings indicate that
miR-19a is a better plasma marker for ESCC detection than Cyfra21-1.

The plasma level of miR-19a in ESCC group and benign lesion group were both increased. Therefore, we next assessed whether the plasma level of miR-19a 
can be used for detection of esophageal tumor (ESCC, esophageal intraepithelial neoplasia, esophageal leiomyomata and esophageal polyps). As shown in Figure 2E, the AUC for miR-19a in esophageal tumor and healthy controls was 0.767 (95\% CI: $0.700-0.835)$. The results showed a high sensitivity (89.34\%) but a low specificity (52.50\%; Table 3). Consistently, the AUC, sensitivity, NPV and diagnosis efficiency of Cyfra21-1 were much lower than those of miR-19a, but the specificity $(87.50 \%)$ was higher (Figure 2F \& Table 3).

High plasma miR-19a levels are complementary to the use of Cyfra21-1 for diagnosis

We next examined whether a combination of $m i R$ $19 a$ and Cyfra21-1 was more sensitive than either marker when used individually. Our analysis showed that they were indeed complementary. In 89 patients with ESCC, miR-19a identified 40 patients that were missed by Cyfra21-1 alone and Cyfra21-1 identified 12 ESCC cases that were missed by miR-19a alone (Figure 3A \& B). By logistic regression of combining with $m i R-19 a$ and Cyfra21-1, the sensitivity was increased to $70.80 \%$, the specificity was $71.20 \%$ and AUC was 0.782 in distinguishing ESCC patients and noncancer controls. With parallel interpretation, the combination of miR-19a and Cyfra21-1 achieved higher sensitivity of $77.27 \%$ and the combination in serial interpretation was able to boost the specificity to $95.16 \%$ (Table 2).

In 47 patients with early stages of ESCC, Cyfra21-1 was detected in 9 patients and the sensitivity was only $19.15 \%$. In contrast, plasma $m i R-19 a$ was detected in 32 patients $(68.09 \%)$ (Figure 3D \& E). For the ability to distinguish early stages of ESCC from noncancer controls, logistic regression of the combined analyses of miR-19a and Cyfra21-1 showed higher AUC (0.758) and sensitivity (78.70\%) but the specificity dropped to $64.80 \%$ (Figure 3F \& Table 2). Consistently, serial testing of miR-19a and Cyfra21-1 dramatically increased the diagnostic specificity $(95.16 \%)$ and the use of parallel testing showed sensitivity of $74.20 \%$.

In 122 patients with esophageal tumor, miR$19 a$ identified 79 patients that were missed by Cyfra21-1 alone and Cyfra21-1 identified 5 esophageal tumor cases that were missed by $m i R-19 a$ alone (Figure 3G \& H). By logistic regression of combination with miR-19a and Cyfra21-1, the AUC reached 0.807 (Figure 31 \& Table 3). With parallel interpretation, the combination of miR-19a and Cyfra21-1 achieved the higher sensitivity of $92.37 \%$ and the combination in serial interpretation was able to boost the specificity to $94.06 \%$ (Table 3). Together, these results indicate that the plasma level of miR-19a combined with Cyfra21-1 provides high diagnosis values and further improves the accuracy of detection.

\section{Downregulation of miR-19a after removal of the primary tumor}

In order to determine whether the increased amount of miR-19a originated from cancer cells and had prognosis value for clinical improvement after surgery, the levels of miR-19a in matched plasma samples $(\mathrm{n}=$ 30 patients) collected before and after surgical resection of primary tumors were compared. As shown in Figure 4A, expressions of miR-19a in 25 out of 30

Table 2. The diagnostic value between esophageal squamous cell carcinoma/early stages of esophageal squamous cell carcinoma and noncancer control.

\begin{tabular}{|c|c|c|c|c|c|}
\hline \multirow{2}{*}{$\begin{array}{l}\text { Variables } \\
\text { Cutoff }\end{array}$} & \multirow{2}{*}{$\begin{array}{l}\text { miR-19a } \\
0.2909\end{array}$} & \multirow{2}{*}{$\begin{array}{l}\text { Cyfra21-1 } \\
3.39 \mathrm{ng} / \mathrm{ml}\end{array}$} & \multicolumn{3}{|c|}{ Combination diagnosis } \\
\hline & & & Logistic regression & Serial testing & Parallel testing \\
\hline \multicolumn{6}{|c|}{ ESCC and noncancer control } \\
\hline Sensitivity (\%) & 66.29 & 32.58 & 70.80 & 21.60 & 77.27 \\
\hline Specificity (\%) & 66.40 & 85.60 & 71.20 & 95.16 & 56.84 \\
\hline PPV (\%) & 58.42 & 61.70 & 63.64 & 76.07 & 56.04 \\
\hline NPV (\%) & 73.45 & 64.07 & 77.40 & 63.03 & 77.84 \\
\hline Diagnosis efficiency (\%) & 66.35 & 63.55 & 71.03 & 64.57 & 65.34 \\
\hline \multicolumn{6}{|c|}{ Early stages of ESCC and noncancer control } \\
\hline Sensitivity (\%) & 68.09 & 19.15 & 78.70 & 13.04 & 74.20 \\
\hline Specificity (\%) & 66.40 & 85.60 & 64.80 & 95.16 & 56.84 \\
\hline PPV (\%) & 43.25 & 33.33 & 45.67 & 50.33 & 39.26 \\
\hline NPV (\%) & 84.70 & 73.79 & 89.00 & 74.43 & 85.42 \\
\hline Diagnosis efficiency (\%) & 66.86 & 67.44 & 68.60 & 72.72 & 61.58 \\
\hline
\end{tabular}


Table 3. The diagnosis value between esophageal tumor and healthy control.

\begin{tabular}{|c|c|c|c|c|c|}
\hline \multirow{2}{*}{$\begin{array}{l}\text { Variables } \\
\text { Cutoff }\end{array}$} & \multirow{2}{*}{$\begin{array}{r}\operatorname{miR}-19 a \\
-0.0305\end{array}$} & \multirow{2}{*}{$\begin{array}{l}\text { Cyfra21-1 } \\
3.39 \mathrm{ng} / \mathrm{ml}\end{array}$} & \multicolumn{3}{|c|}{ Combination diagnosis } \\
\hline & & & $\begin{array}{l}\text { Logistic } \\
\text { regression }\end{array}$ & Serial testing & Parallel testing \\
\hline Sensitivity (\%) & 89.34 & 28.69 & 73.80 & 25.61 & 92.37 \\
\hline Specificity (\%) & 52.50 & 87.50 & 73.80 & 94.06 & 45.94 \\
\hline PPV (\%) & 74.15 & 77.78 & 81.12 & 86.80 & 72.27 \\
\hline NPV (\%) & 76.36 & 44.59 & 64.88 & 45.33 & 79.79 \\
\hline $\begin{array}{l}\text { Diagnosis } \\
\text { efficiency (\%) }\end{array}$ & 74.75 & 51.98 & 73.80 & 52.72 & 73.98 \\
\hline
\end{tabular}

patients were decreased $(\mathrm{p}<0.001)$ after surgery. The results suggest that miR-19a may be secreted by cancer cells and that the level of miR-19a may reflect the ESCC status of the patient.

In the follow-up studies, one of the five patients that still exhibited high levels of miR-19a $(1 / 5=20 \%)$ developed liver and lung metastasis in 10 months later after surgery. In contrast, only one from the patients that exhibited decreased miR-19a expression (1/25 $=4 \%$ ) developed lymph node metastasis (Figure 4B). Together, the data suggest that the postoperative metastasis probability in patients with a high $m i R$ $19 a$ level was much higher than in the downregulated group, demonstrating that the plasma level of miR-19a is of potential prognosis value for ESCC progression after surgery. Whether there is a relationship between the postoperative expressions of $m i R-19 a$ and distant metastasis requires additional clinical data analysis.

\section{Preoperational miR-19a level in patients diagnosed with recurrence/metastasis after surgery}

To estimate whether plasma miR-19a can predict recurrence/metastasis in patients with ESCC after surgery, the miR-19a expression and patient history from 49 ESCC patients that had surgery (49 of 89 ESCC patients) were analyzed. The results showed that the preoperative levels of $m i R-19 a$ in patients with recurrence/metastasis 10 months after surgery $(n=6)$ was much higher than in patients without recurrence/ metastasis after surgery $(\mathrm{n}=43$; Figure 5$)$, suggesting that plasma miR-19a level may be a valuable predictor of postoperative recurrence probability.

\section{Discussion}

$m i R-19 a$ is located in the miR-17-92 cluster and was previously reported to be highly expressed in ESCC tissues [16,26]. Herein, we reported that expression of miR-19a in the plasma of ESCC patients was increased and changes in $m i R-19 a$ plasma level predicted the risk of postsurgery metastasis of ESCC patients. These findings support the use of plasma miR-19a levels as a biomarker for ESCC. To our knowledge, this is the first comprehensive study to measure miR-19a expression and assess its clinical significance for ESCC patients.

Clinical data showed that for patients in the early stages of ESCC, the 5-year and 10-year survival rate after resection could reach more than 85.9 and $55.6 \%$, respectively [28]. However, due to the lack of obvious symptoms in the early stages of ESCC and the lack of a high sensitive biomarker allowing early diagnosis, the majority of patients with ESCC are only diagnosed when the cancer reaches an advanced stage, resulting in a 5-year postsurgery survival rate of only $20-40 \%$ [29,30]. Therefore, improved biomarkers that allow early ESCC detection are urgently needed. In this study, miR-19a levels were similarly increased in ESCC patients and patients with benign lesions compared with healthy controls. miR-19a levels showed high sensitivity for detection in early stages of ESCC and esophagus tumor. According to the ROC, the AUC could reach 0.729 and 0.767 , respectively. The sensitivity and specificity were 68.09 and $66.40 \%$ in distinguishing the early stages of ESCC. In distinguishing the esophagus tumor from healthy controls, the sensitivity and specificity were 89.34 and $52.50 \%$. These findings validated the promise of using plasma miR-19a as a biomarker for early screening of high-risk individuals for ESCC, which is critical for early prevention and treatment to improve outcomes.

Currently, Cyfra21-1 is used as a plasma biomarker for ESCC detection. However, Cyfra21-1 exhibits a low sensitivity in detection of ESCC $[10,31,32]$. Our analysis also showed that the sensitivity of Cyfra21-1 in ESCC and in the early stages of ESCC detection was only 32.58 and $19.15 \%$, respectively, but the use of miR-19a together with Cyfra21-1 allowed improved sensitivity. Logistical regression combined with miR-19a and Cyfra 21-1 provided high diagnosis value and further improved the accuracy of detection. Additionally, combination of miR-19a and Cyfra21-1 in parallel achieved 
(A)

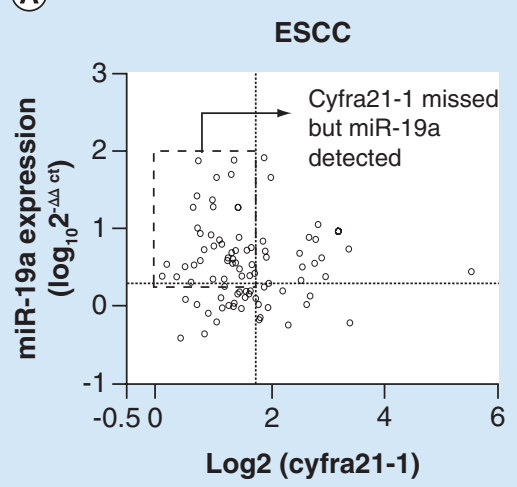

(D)

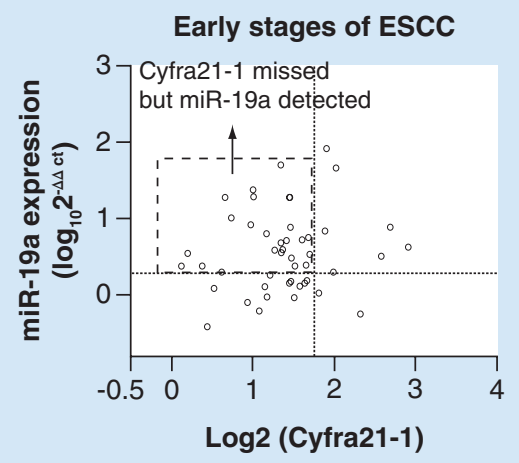

(G)

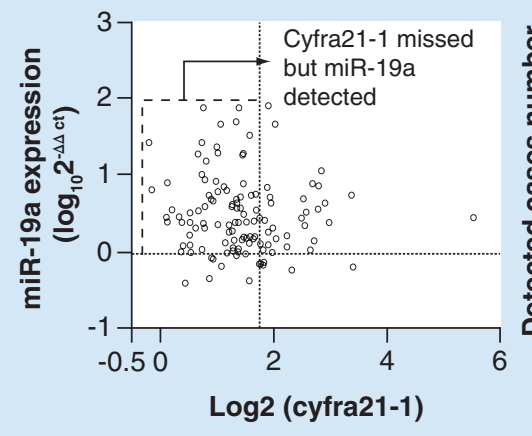

(B)

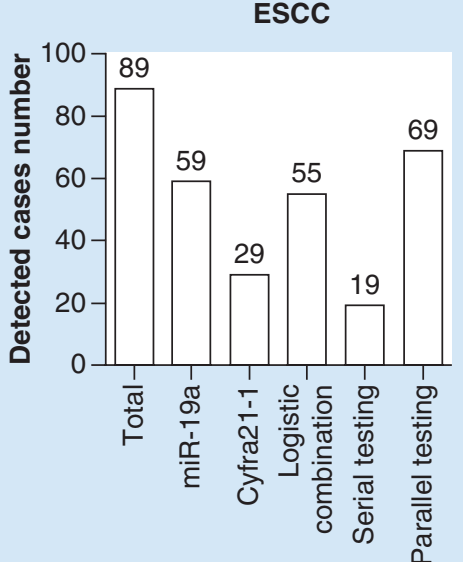

(E)

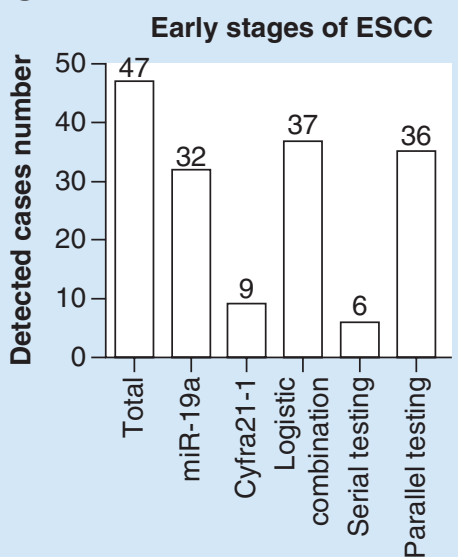

(H)

Esophageal tumor

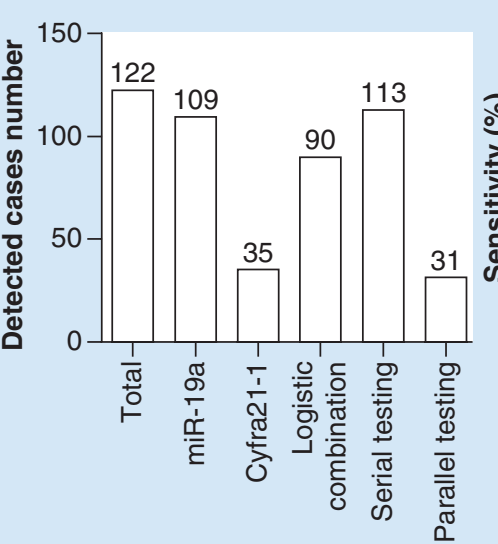

(C)

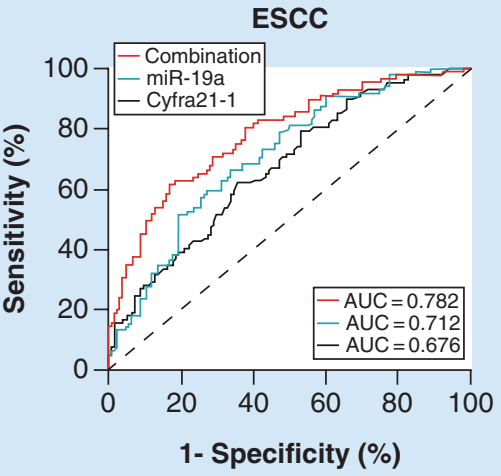

(F)

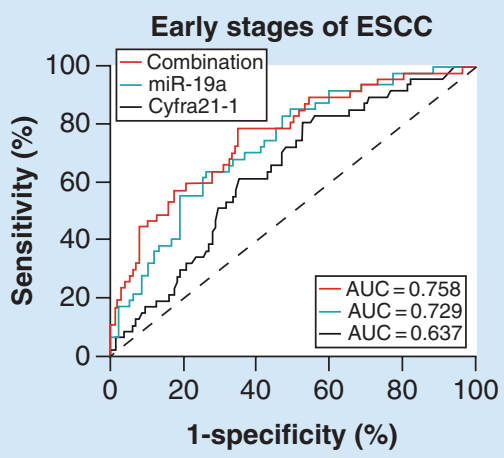

(1)

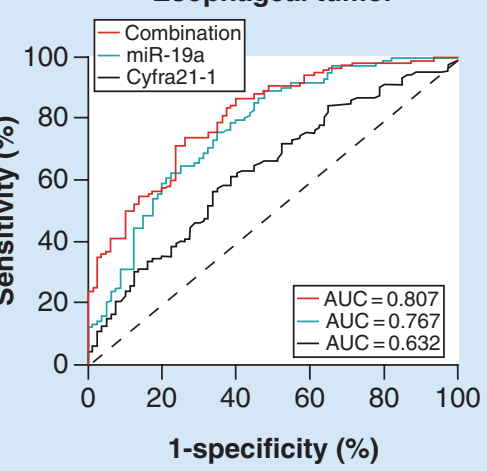

Figure 3. Combination of miR-19a and Cyfra21-1 have higher diagnosis values. (A, D, G) Two-parameter classification is used to detect ESCC, early stages of ESCC or esophageal tumor. The cut-off value for miR-19a is calculated from the ROC curve. The cut-off value for Cyfra21-1 is presented as $\log _{2}$. (B, E, H) Detection rates of miR-19a, Cyfra21-1 and combination of miR-19a and Cyfra21-1 in patients with ESCC, early stages of ESCC or esophageal tumor. (C, F, I) Combination ROC curve analyses of miR-19a and Cyfra21-1 for patients with ESCC, early stages of ESCC and noncancer controls or esophageal tumor and healthy controls.

Cyfra: Cytokeratin 19 fragment; ESCC: Esophageal squamous cell carcinoma; ROC: Receiver operating characteristic. 
(A)

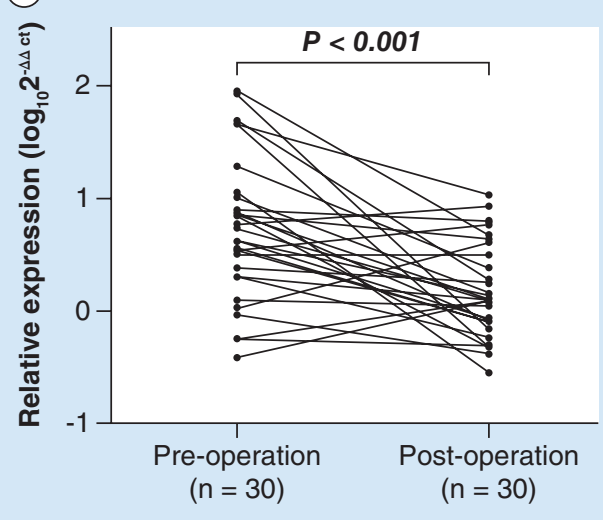

(B)

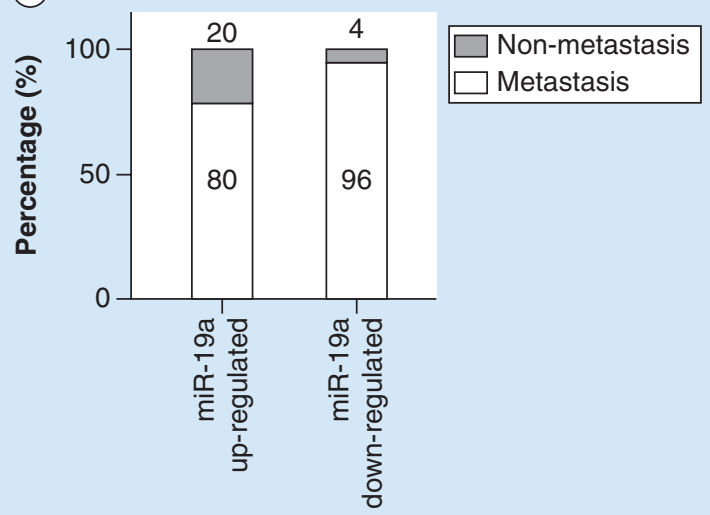

Figure 4. Prognosis value of $m i R-19 a$ for clinical improvement after surgery.(A) The plasma level of $m i R-19 a$ before (preoperation) and after surgery (postoperation). The $y$-axis indicates the relative values of $m i R-19 a$ which were normalized to cel-miR-39 and shown as $\log _{10}$ (Wilcoxon matched-paired signed ranks sum test).

(B) The percentage of patients with metastasis 10 months after the surgery in miR-19a upregulated group and downregulated group were compared.

higher sensitivity for early diagnosis and screening of ESCC. Serial testing dramatically increased the diagnostic specificity and PPV, allowing identification of false positive results to avoid unnecessary treatment. Taken together, these findings clearly demonstrated that plasma miR-19a combined with Cyfra21-1 can be used as effective plasma biomarkers for ESCC.

Our analysis showed that high levels of plasma miR$19 a$ predicted poor survival and thus $m i R-19 a$ was an independent prognostic factor for advanced ESCC. Our data also revealed that $m i R-19 a$ was significantly downregulated after the surgical resection of primary tumors, thus providing a useful insight into the application of plasma miR-19a in the evaluation of therapeutic effect. It has been reported that circulating miRNA is not only derived from blood cells but also from the tissues affected by disease such as tumor tissues [25,33], suggesting that the deregulated miR-19a plasma levels in ESCC patients could be secreted actively or passively by tumor cells. Interestingly, the data suggest that the postoperative metastasis probability in upregulated patients was much higher than for the patients in the postoperative downregulated group, indicating that the plasma levels of $m i R$ $19 a$ may be of potential prognosis values for ESCC progression after surgery. Because of the high recurrence and metastasis rates after surgery, the 5-year survival rate of ESCC treated with surgery alone is poor, only approximately $25 \%$ [34]. Thus, it is urgent to find a biomarker that can well predict ESCC metastasis/recurrence. Interestingly, our data showed that the preoperative level of $m i R-19 a$ in patients with recurrence/metastasis after surgery was significantly higher than patients who did not have recurrence/metastasis after surgery, suggesting that $m i R-19 a$ is a promising biomarker for ESCC recurrence/metastasis surveillance. Further studies with higher number of patients in each cancer stage category and higher number with benign conditions are needed to validate these findings.

\section{Conclusion}

In conclusion, plasma miR-19a may represent a novel biomarker that complements Cyfra21-1 for the detection of ESCC. Plasma miR-19a may be potentially useful for cancer screening, monitoring ESCC prognosis postoperatively and predicting recurrence/metastasis after surgery.

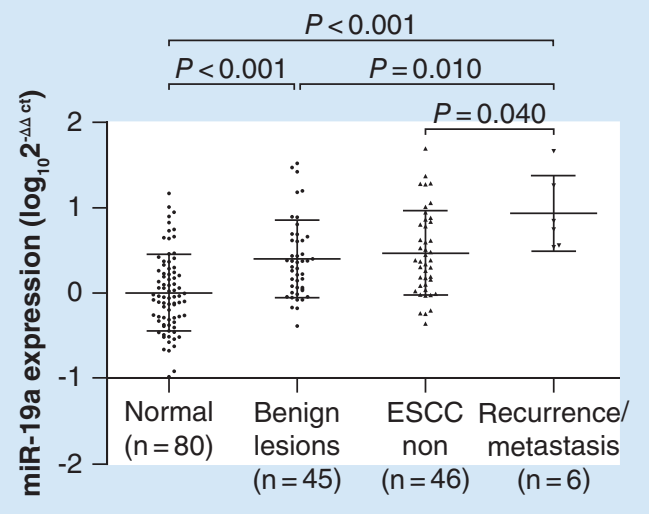

Figure 5. Preoperational miR-19a level in patients diagnosed with recurrence/metastasis after surgery. The relative levels of miR-19a in patients with benign lesions, ESCC and healthy controls. The y-axis indicates the relative values of miR-19a which were normalized to cel-miR-39 and shown as $\log _{10}$.

ESCC: Esophageal squamous cell carcinoma. 
Supplementary data

To view the supplementary data that accompany this paper please visit the journal website at: www.futuremedicine.com/ doi/full/10.2217/bmm-2016-0286

\section{Author contributions}

$Y$ Bai and Z Fang participated in the experiment designs and carried out the experiment. Y Bai and $\mathrm{H}$ Lin drafted the manuscript. $\mathrm{H}$ Lin and Q Luo performed the statistical analysis. Y Fang, Y Su and Q Hu helped to collect the plasma samples and clinical data of patients. $\mathrm{H}$ Duan and $\mathrm{F}$ Chen revised the manuscript. Z-Y Zhang designed the experiment and revised the manuscript. All authors read and approved the final manuscript.

\section{Open access}

This work is licensed under the Attribution-NonCommercialNoDerivatives 4.0 Unported License. To view a copy of this license, visit http://creativecommons.org/licenses/by-nc-nd/4.0/
Financial \& competing interests disclosure

This work was supported by the National Natural Science Foundation of China (grant numbers 81401971, 81311120473, 81072016), Postdoctoral Science Foundation of China (grant number 2015T80682). The authors have no other relevant affiliations or financial involvement with any organization or entity with a financial interest in or financial conflict with the subject matter or materials discussed in the manuscript apart from those disclosed.

No writing assistance was utilized in the production of this manuscript.

Ethical conduct of research

The authors state that they have obtained appropriate institutional review board approval or have followed the principles outlined in the Declaration of Helsinki for all human experimental investigations. In addition, for investigations involving human subjects, informed consent has been obtained from the participants involved.

\section{Summary points}

- Early detection is critical to improve outcomes and reduce mortality of esophageal squamous cell carcinoma (ESCC) patients. Currently, the sensitivity and validity of commonly used circulating biomarkers are not sufficient for ESCC detection, particularly at early stages.

- miR-19a remains stable for a long period of time even under harsh conditions.

- The abundance of miR-19a in the plasma was significantly increased in patients with benign lesions and ESCC.

- The sensitivity and specificity of miR-19a for ESCC detection was 66.29 and $66.40 \%$ (area under the curve $(A \cup C)=0.712$ ). cytokeratin 19 fragment (Cyfra) 21-1, the commonly used diagnosis marker for ESCC, showed a lower AUC (0.676) than miR-19a for ESCC detection.

- The sensitivity and specificity of miR-19a were 68.09 and $66.40 \%$ in distinguishing early stages of ESCC from noncancer controls ( $A \cup C=0.729$ ). The AUC, sensitivity, positive predictive value and negative predictive value of Cyfra21-1 were much lower than miR-19a for the diagnosis of early stages of ESCC.

- The sensitivity and specificity of miR-19a for esophageal tumor detection was 89.34 and $52.50 \%$ (AUC =0.767). The AUC, sensitivity, negative predictive value and diagnosis efficiency of Cyfra21-1 were much lower than miR-19a, but the specificity $(87.50 \%)$ was much higher.

- High plasma miR-19a levels complement with Cyfra21-1 in diagnosis of ESCC, early stages of ESCC and esophageal tumor. Assaying plasma miR-19a levels combined with Cyfra21-1 improved the successful diagnosis of ESCC, particularly at early stages of ESCC and esophageal tumor.

- Expressions of miR-19a in $83.33 \%$ patients was decreased after surgery and the patients with increased miR-19a had a high risk of metastasis, suggesting that changes in plasma level of miR-19a after surgery has predictive value for postoperative metastasis.

\section{References}

Papers of special note have been highlighted as:

- of interest; $\bullet \bullet$ of considerable interest

1 Zhang C, Wang C, Chen X et al. Expression profile of microRNAs in serum: a fingerprint for esophageal squamous cell carcinoma. Clin. Chem. 56(12), 1871-1879 (2010).

2 Wobst A, Audisio RA, Colleoni M, Geraghty JG. Oesophageal cancer treatment: studies, strategies and facts. Ann. Oncol. 9(9), 951-962 (1998).

3 Guo Y, Chen Z, Zhang L et al. Distinctive microRNA profiles relating to patient survival in esophageal squamous cell carcinoma. Cancer Res. 68(1), 26-33 (2008).
4 Torre LA, Bray F, Siegel RL, Ferlay J, Lortet-Tieulent J, Jemal A. Global cancer statistics, 2012. CA Cancer J. Clin. 65(2), 87-108 (2015).

5 Yuequan J, Shifeng C, Bing Z. Prognostic factors and family history for survival of esophageal squamous cell carcinoma patients after surgery. Ann. Thorac. Surg. 90(3), 908-913 (2010).

6 Chen J, Pan J, Zheng X et al. Number and location of positive nodes, postoperative radiotherapy, and survival after esophagectomy with three-field lymph node dissection for thoracic esophageal squamous cell carcinoma. Int. J. Radiat. Oncol. Biol. Phys 82(1), 475-482 (2012). 
$7 \quad$ Pisani P, Parkin DM, Bray F, Ferlay J. Estimates of the worldwide mortality from 25 cancers in 1990. Int. J. Cancer 83(1), 18-29 (1999).

8 Roulston JE. Limitations of tumour markers in screening. $B r$. J. Surg. 77(9), 961-962 (1990).

9 Duffy MJ. Role of tumor markers in patients with solid cancers: a critical review. Eur. J. Intern. Med. 18(3), 175-184 (2007).

- $\quad$ Summarizes the basic knowledge about cytokeratin 19 fragment 21-1.

10 Yamamoto K, Oka M, Hayashi H, Tangoku A, Gondo T, Suzuki T. CYFRA 21-1 is a useful marker for esophageal squamous cell carcinoma. Cancer 79(9), 1647-1655 (1997).

- Summarizes the basic knowledge about squamous cell carcinoma antigen and other markers in esophageal squamous cell carcinoma.

11 Mealy K, Feely J, Reid I, Mcsweeney J, Walsh T, Hennessy TP. Tumour marker detection in oesophageal carcinoma. Eur. J. Surg. Oncol. 22(5), 505-507 (1996).

12 Ambros V. The functions of animal microRNAs. Nature 431(7006), 350-355 (2004).

13 Kloosterman WP, Plasterk RH. The diverse functions of microRNAs in animal development and disease. Dev. Cell 11(4), 441-450 (2006).

14 Stefani G, Slack FJ. Small non-coding RNAs in animal development. Nat. Rev. Mol. Cell Biol. 9(3), 219-230 (2008).

15 Calin GA, Sevignani C, Dumitru CD et al. Human microRNA genes are frequently located at fragile sites and genomic regions involved in cancers. Proc. Natl Acad. Sci. USA 101(9), 2999-3004 (2004).

-. Shows the role of miR-19a in esophageal squamous cell carcinoma.

16 Xu XL, Jiang YH, Feng JG, Su D, Chen PC, Mao WM. MicroRNA-17, microRNA-18a, and microRNA-19a are prognostic indicators in esophageal squamous cell carcinoma. Ann. Thorac. Surg. 97(3), 1037-1045 (2014).

17 Yang C, Ning S, Li Z, Qin X, Xu W. miR-22 is downregulated in esophageal squamous cell carcinoma and inhibits cell migration and invasion. Cancer Cell Int. 14(1), 138 (2014).

18 Zhang BJ, Gong HY, Zheng F, Liu DJ, Liu HX. Upregulation of miR-335 predicts a favorable prognosis in esophageal squamous cell carcinoma. Int. J. Clin. Exp. Pathol. 7(9), 6213-6218 (2014).

19 Ding J, Huang S, Wu S et al. Gain of miR-151 on chromosome 8q24.3 facilitates tumour cell migration and spreading through downregulating RhoGDIA. Nat. Cell Biol. 12(4), 390-399 (2010).

20 Meng Z, Fu X, Chen X et al. miR-194 is a marker of hepatic epithelial cells and suppresses metastasis of liver cancer cells in mice. Hepatology 52(6), 2148-2157 (2010).

21 Hou J, Lin L, Zhou Wet al. Identification of miRNomes in human liver and hepatocellular carcinoma reveals miR-
$199 \mathrm{a} / \mathrm{b}-3 \mathrm{p}$ as therapeutic target for hepatocellular carcinoma. Cancer Cell 19(2), 232-243 (2011).

22 Gregory PA, Bert AG, Paterson EL et al. The miR-200 family and miR-205 regulate epithelial to mesenchymal transition by targeting ZEB1 and SIP1. Nat. Cell Biol. 10(5), 593-601 (2008).

23 Shimono Y, Zabala M, Cho RW et al. Downregulation of miRNA-200c links breast cancer stem cells with normal stem cells. Cell 138(3), 592-603 (2009).

24 Bohlig L, Friedrich M, Engeland K. p53 activates the PANK1/miRNA-107 gene leading to downregulation of CDK6 and p130 cell cycle proteins. Nucleic Acids Res. 39(2), 440-453 (2011).

-. Summarizes the role of circulating miRNA as markers for cancer detection.

25 Mitchell PS, Parkin RK, Kroh EM et al. Circulating microRNAs as stable blood-based markers for cancer detection. Proc. Natl Acad. Sci. USA 105(30), 10513-10518 (2008).

- Shows the mechanism of cancer development regulated by miR-19a.

26 Liu M, Wang Z, Yang S et al. TNF-alpha is a novel target of miR-19a. Int. J. Oncol. 38(4), 1013-1022 (2011).

27 Fang Z, Tang J, Bai Y et al. Plasma levels of microRNA-24, microRNA-320a, and microRNA-423-5p are potential biomarkers for colorectal carcinoma. J. Exp. Clin. Cancer Res. 34, 86 (2015).

28 Huang GJ. Early detection and surgical treatment of esophageal carcinoma. Jpn J. Surg. 11(6), 399-405 (1981).

29 Ando N, Ozawa S, Kitagawa Y, Shinozawa Y, Kitajima M. Improvement in the results of surgical treatment of advanced squamous esophageal carcinoma during 15 consecutive years. Ann. Surg. 232(2), 225-232 (2000).

30 Mariette C, Finzi L, Fabre S, Balon JM, Van Seuningen I, Triboulet JP. Factors predictive of complete resection of operable esophageal cancer: a prospective study. Ann. Thorac. Surg. 75(6), 1720-1726 (2003).

- Shows the clinical significance of serum cytokeratin 19 fragment 21-1 for esophageal cancer.

31 Brockmann JG, St Nottberg H, Glodny B, Heinecke A, Senninger NJ. CYFRA 21-1 serum analysis in patients with esophageal cancer. Clin. Cancer Res. 6(11), 4249-4252 (2000).

32 Brockmann JG, St Nottberg H, Glodny B, Sprakel B, Senninger N. Analysis of serum CYFRA 21-1 concentrations in patients with esophageal cancer. Anticancer Res. 20(6D), 4899-4904 (2000).

33 Chen X, Ba Y, Ma L et al. Characterization of microRNAs in serum: a novel class of biomarkers for diagnosis of cancer and other diseases. Cell Res. 18(10), 997-1006 (2008).

34 Mariette C, Piessen G, Triboulet JP. Therapeutic strategies in oesophageal carcinoma: role of surgery and other modalities. Lancet Oncol. 8(6), 545-553 (2007). 